\title{
Proton Hyperfine Splitting Constants of Azulene Cation in Accordance with some Modifications of the McLachlan Method
}

\author{
J. NowAKOWSKI \\ Department of Theoretical Chemistry, Silesian University, Katowice, Poland
}

(Z. Naturforsch. 28 a, 233-235 [1973]; received 31 October 1972)

\begin{abstract}
The accuracy of a theoretical prediction of the proton hyperfine splitting constants of the azulene cation has been analyzed with the use of the McConnell relationship. Spin densities have been calculated with various modifications of the McLachlan method. The results have been compared with experimental data and with predictions of other authors.
\end{abstract}

The McLachlan method 1 and the McConnell relationship 2 are commonly used when interpreting the EPR spectrum of $\pi$-electron radicals. In the McLACHLAN scheme ${ }^{1}$ a semiempirical parameter $\lambda$ appears which according to McLachlan is equal to 1.0-1.2. However, it was proved most recently for a large group of ionic radicals of alternant and nonalternant hydrocarbons ${ }^{3}$ that the value $\lambda=0.6$ gives much better results. and for a cation radical that

$$
\varrho_{\mu}=c_{0 \mu}^{2}(0)+\sum_{j}^{\text {doubly occ. }}\left[c_{j \mu}^{2}(0)-c_{j \mu}^{2}(-2 \lambda)\right]
$$

where the LCAO coefficients $c_{j \mu}( \pm 2 \lambda)$ 's follow from a diagonalization of the perturbed energy matrix such that

$$
H_{\mu v}( \pm 2 \lambda)=H_{\mu \nu} \pm 2 \lambda c_{0 \mu}^{2}(0) \delta_{\mu \nu} \beta^{0}
$$

\begin{tabular}{|c|c|c|c|c|}
\hline position $^{a}$ & experimental $^{\mathrm{b}}$ & $\begin{array}{l}\text { Hückel method } \\
\beta_{9-10}=0.9 \beta^{\circ}\end{array}$ & $\begin{array}{l}\text { McLachlan method } \\
\text { Ref. } 6\end{array}$ & $\begin{array}{l}\text { McLachlan method } \\
\text { present work }\end{array}$ \\
\hline 1 & 2 & 3 & 4 & 5 \\
\hline $\begin{array}{l}1 \\
2 \\
4 \\
5 \\
6\end{array}$ & $\begin{array}{l}0.394 \\
0.056 \\
0.014 \\
0.154 \\
0.041\end{array}$ & $\begin{array}{l}0.284 \\
0 \\
0.029 \\
0.119 \\
0\end{array}$ & $\begin{array}{r}0.293 \\
-0.010 \\
0.024 \\
0.120 \\
-0.005\end{array}$ & $\begin{array}{r}0.398 \\
-0.102 \\
0.005 \\
0.138 \\
-0.048\end{array}$ \\
\hline
\end{tabular}

Table 1. Spin densities in azulene cation radical.

a See Figure 1.

b Absolute values calculated from the McConnell relationship with $Q=-27 \mathrm{G}$, using experimental proton hyperfine splitting constants listed in column 2 of Table 26 .

c With atom-atom polarizabilities calculated from H MO's $\left(\beta_{9-10}=0.9 \beta^{\circ}\right) ; \lambda=1.2$.

Let us also recall that a useful modification of the McLachlan method has been recently derived for neutral radicals of odd alternant hydrocarbons ${ }^{4}$. The modification can be extended to ionic radicals. Let $c_{j \mu}(0)$ be the expansion coefficient of the j-th molecular orbital $(j=0$ denotes singly occupated molecular orbital) in terms of the $2 p \pi$ atomic orbital of atom $\mu$, in accordance with the selfconsistent Hückel method, $\mathrm{SCH} \mathrm{MO}^{5}$. It follows then from a perturbational treatment of the UHF method for a neutral or anion radical that

$$
\varrho_{\mu}=c_{0 \mu}^{2}(2 \lambda)+\sum_{j}^{\text {doubly occ. }}\left[c_{j \mu}^{2}(2 \lambda)-c_{j \mu}^{2}(0)\right]
$$

Reprint requests to Dr. J. NowAKowSKI, Department of Theoretical Chemistry, Institute of Chemistry, Silesian University, Szkolna 9, Katowice, Poland.
In the case of neutral radicals of odd alternant hydrocarbons the modified MacLachlan formula (1) yielded a definitely better agreement with experiment than the original one 4 .

Many authors believe the McLachlan method to be ineffective in the case of ionic radicals of nonalternant hydrocarbons. Such an opinion was formulated, for example, most recently by DesSAU and SHIH ${ }^{6}$ in their discussion of the EPR spectrum of the azulene cation radical. The authors have determined the proton hyperfine splitting constants for this radical claiming the McLachlan to provide a negligible improvement of the simple Hückel method.

The purpose of this work is to show that such an opinion is not grounded being partly based on erroneous numerical results. It is also shown that the modified 


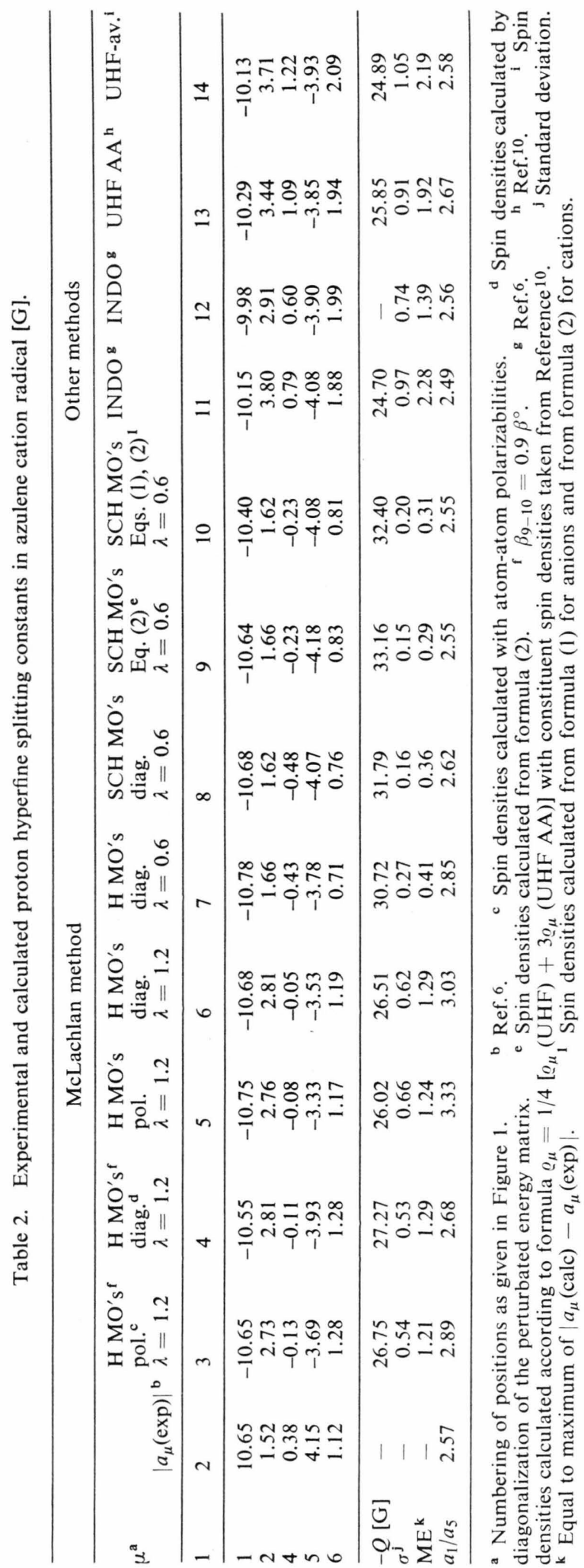

treatments (1) and (2) with $\lambda=0.6$ yield again results which are significantly better than those obtained in other treatments.

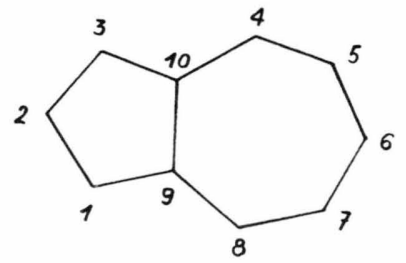

Fig. 1. Numbering of positions.

In Table 1 we compare the spin densities for the azulene cation as following from the experiment (column 2), the simple Hückel method with $\beta_{9-10}=0.9$ $\beta 06,7$ (column 3), the $\mathrm{H} \mathrm{MO}$ based perturbational McLachlan method with $\lambda=1.2$ and $\beta_{9-10}=0.9 \beta 0$, as quoted erroneously by Dessau and SHIH 6 (column 4). In the column 5 we quote the recalculated values. One notes that correct values show a significant improvement over the simple Hückel method in contrast to the statement of Dessau and Shih.

In Table 2 we compare the experimental hyperfine splitting constants 6 (column 2) with the calculated ones in accordance with various modifications of the McLachlan method and with more advanced treatments. The McConnell relationship $a_{\mu}=Q \varrho_{u}$ has been used with this purpose. Except the case reported in column 10 the value of $Q$ has been fitted with a least squares method for all experimental points of the azulene cation. In the case of column 10 the $Q$ value is optimized for a set of 10 ionic radicals of nonalternant hydrocarbons, $Q=-32.40 \mathrm{G}$.

In the table we quote also the standard deviation $(\sigma)$, the maximal error (ME) and the ratio of the hyperfine splitting constants $a_{1} / a_{5}$.

Let use note that differences resulting from the two alternative methods of calculation of spin densities proposed by McLaChLAN ${ }^{8}$ (columns 3 and 4 or 5 and 6) are comparable to the correction of spin density following by a reduction of the resonance integral $\beta_{9-10}$ (columns 3 and 5 or 4 and 6) as suggested by BerNAL, Rieger and Fraenkel ${ }^{7}$ and Dessau and Shih ${ }^{6}$. Therefore the correction must be considered as insignificant.

It follows from Table 2 that a large improvement is obtained within the McLachlan method taking for $\lambda 0.6$ (columns 7-9) instead of 1.2 (columns 3-6). A farther improvement is achieved by replacing the $\mathrm{H} \mathrm{MO}$ basis (column 7) by the SCH MO one (columns 8-9). Additional small improvement is obtained in the case of the modified McLachlan method.(2) (column 9).

Results which have been obtained with more advanced treatments are worse. In columns 11 and 12 we quote those obtained with the INDO method for the experimental X-ray structure ${ }^{9}$. Column 11 refers to an 
indirect calculation of the splitting constants and column 12 to the direct one. Column 13 gives the results of the UHF method with anihilation of unwanted multiplicity (UHF AA) within the PPP scheme ${ }^{10}$. Column 14 refers to a modification suggested by AMOS and SNY$\mathrm{DER}^{11}$, basing on an averaging process of the results of the UHF and UHF AA methods. In yielded good results for a number of radicals $12-15$. We do not analyze the results of other authors which are in a worse agreement with experiment $16-20$.

As follows from this comparison the direct calculation within the INDO scheme yields best results for all advanced treatments. They are, however, still significantly worse than those following from the self consistent Hückel method and the modified McLachlan treatment with $\lambda=0.6$.

1 A. D. Mclachlan, Mol. Phys. 3, 233 [1960].

2 H. M. McConnell, J. Chem. Phys. 24, 764 [1956].

3 J. Nowakowski, Chem. Phys. Letters 2, 289 [1968].

4 J. Nowakowski, Theor. Chim. Acta [Berl.] 18, 133 [1970].
5 A. Golebiewski and J. Nowakowski, Acta Phys. Polon. 25, 647 [1964].

6 R. M. Dessau and S. Shih, J. Chem. Phys. 53, 3169 [1970].

7 I. Bernal, P. H. Rieger, and G. K. Fraenkel, J. Chem. Phys. 37, 1498 [1962].

8 S. Kowalski, Thesis, Jagellonian University, Krakow 1971.

9 J. M. Robertson, H. M. M. Shearer, G. A. Sim, and D. G. Watson, Acta Cryst. 15, 1 [1962].

10 A. T. Amos, Mol. Phys. 5, 91 [1962].

11 L. C. SNYDER and T. Amos, J. Chem. Phys. 42, 3670 [1965] and references therein.

12 T. Amos and M. Woodward, J. Chem. Phys. 50, 119 [1969].

13 N. K. Ray and P. T. Narasimhan, Theor. Chim. Acta [Berl.] 8, 306 [1967].

14 A. W. Salotto and L. Burnelle, J. Chem. Phys. 53, 333 [1970].

15 P. Cavalieri D'Oro, R. Danieli, G. Maccagnani, G. F. Pedulli, and P. Palmieri, Mol. Phys. 20, 365 [1971].

16 A. Hinchliffe, Theor. Chim. Acta [Berl.] 5, 208 [1966].

17 J. E. Harriman and K. M. Sando, J. Chem. Phys. 48, 5138 [1968].

18 J. E. Bloor, Can. J. Chem. 43, 3026 [1965].

19 T. H. Brown, J. Chem. Phys. 41, 2223 [1964].

20 G. Roberts and K. Warren, Theor. Chim. Acta [Berl.] 22, 184 [1971]. 